\title{
PAPERS
}

\section{Relative risk of dysplasia for patients with intestinal metaplasia in the distal oesophagus and in the gastric cardia}

\author{
P Sharma, A P Weston, T Morales, M Topalovski, M S Mayo, R E Sampliner
}

\begin{abstract}
Background-Biopsy specimens obtained from the gastro-oesophageal junction can reveal intestinal metaplasia in patients presenting for routine upper endoscopy. The site of biopsy may play a critical role in determining the dysplasia risk of a patient.

Aims-To evaluate prospectively the dysplasia risk in patients with intestinal metaplasia of the distal oesophagus or within the gastric cardia.

Methods-Patients with short segment Barrett's oesophagus (SSBO) and cardia intestinal metaplasia (CIM) were followed prospectively.

Results-177 patients with SSBO were identified (mean age 62 years, range 38-82; 91\% whites). Twenty prevalence cases of dysplasia in SSBO were detected: 17 low grade dysplasia (LGD), three high grade dysplasia (HGD). Seventy six patients with CIM were identified (mean age 67 years, range $37-81 ; 81 \%$ whites). A single prevalence case of LGD in CIM was detected. During follow up of $78 \mathrm{SSBO}$ and 34 CIM patients, dysplasia developed in nine (seven LGD, two HGD) with SSBO and in one (LGD) with CIM. There were significant differences between the two groups with respect to age, ethnicity, dysplasia prevalence, and incidence. Time to dysplasia progression was significantly longer in CIM compared with SSBO patients. Of the five patients with SSBO and HGD, one developed adenocarcinoma of the oesophagus on follow up. No HGD or cancers have been detected over this time period in CIM patients.

Conclusions-The dysplasia risk is significantly greater in SSBO than in CIM patients, indicating two potentially different clinical processes. Future studies should separate SSBO from CIM in order to enhance the understanding of the pathophysiology and malignant potential of each entity. (Gut 2000;46:9-13)
\end{abstract}

Keywords: high grade dysplasia; low grade dysplasia; metaplasia; Barrett's oesophagus; gastro-oesophageal reflux disease
Biopsy specimens obtained from the gastrooesophageal junction (GOJ), in patients undergoing routine upper endoscopy, often reveal intestinal metaplasia. These specimens may have been obtained either from the distal oesophagus or immediately distal to the GOJ - that is, the gastric cardia. The gastric cardia is the most proximal part of the stomach that lies immediately distal to the GOJ. Intestinal metaplasia in the distal oesophagus and gastric cardia has been of great interest recently due to the rapidly rising cancer incidence in these locations. ${ }^{1-3}$ Carcinomas arising from the gastric cardia differ from those in the remainder of the stomach. They share common epidemiological characteristics with oesophageal adenocarcinoma and may originate from segments of intestinal metaplasia in the oesophagus - that is, Barrett's oesophagus. ${ }^{4}$

The traditional definition of Barrett's oesophagus included at least $3 \mathrm{~cm}$ of circumferential columnar lining in the distal oesophagus. ${ }^{5}$ However, the entity of short segment Barrett's oesophagus, defined as segments of intestinal metaplasia less than $3 \mathrm{~cm}$ in length, has been recently defined and well accepted in the literature. ${ }^{6}$ Intestinal metaplasia at the GOJ has been reported to vary from $5 \%$ to $25 \%$ in patients presenting for upper endoscopy. ${ }^{7-9}$ These series included patients with either a normal squamocolumnar junction or short segments of columnar appearing mucosa in the distal oesophagus. However, few studies separate the prevalence of intestinal metaplasia in the distal oesophagus from that in the gastric cardia. ${ }^{10-14}$

Short segment Barrett's oesophagus has been associated with gastro-oesophageal reflux disease (GORD), is typically detected in white men, and has the risk of dysplasia and carcinoma. ${ }^{15} 16$ However, the dysplasia risk of patients with cardia intestinal metaplasia is unclear. The goals of the current study were to define cardia intestinal metaplasia, separate it

Abbreviations used in this paper: CIM, cardia intestinal metaplasia; GOJ, gastro-oesophageal junction; GORD, gastro-oesophageal reflux disease; HGD, high grade dysplasia; LGD, low grade dysplasia; LSBO, long segment Barrett's oesophagus; OGD, oesophagogastroduodenoscopy; SSBO, short segment Barrett's oesophagus.
Accepted for publication 6 July 1999 
Table 1 Clinical characteristics of patients with short segment Barrett's oesophagus (SSBO) and cardia intestinal metaplasia (CIM)

\begin{tabular}{llll}
\hline & SSBO $(n=177)$ & CIM $(n=76)$ & $p$ Value \\
\hline Mean age (y) & 62 & 67 & 0.0045 \\
Sex (M/F) & $176 / 1$ & $75 / 1$ & 0.511 \\
Ethnicity & $91 \%$ & $81 \%$ & \\
$\quad$ Whites & $4 \%$ & $16 \%$ & 0.0028 \\
$\quad$ African Americans & $5 \%$ & $3 \%$ & \\
$\quad$ Others & $11.3 \%$ & $1.3 \%$ & 0.0058 \\
Dysplasia prevalence & $4.6 \%$ & $1.5 \%$ & $0.0077^{\star}$ \\
Dysplasia incidence (per year) & 5 & 0 & 0.326 \\
No of patients with high grade dysplasia & 1 & 0 & 1.000 \\
No of patients with cancer & 1 & &
\end{tabular}

^Time to dysplasia development per log rank test.

from short segment Barrett's oesophagus, and prospectively follow these patients for the development of dysplasia and adenocarcinoma.

\section{Methods}

PATIENT POPULATION

Patients were enrolled from the Kansas City VA Medical Center and Tucson VA Medical Center. The study was approved by the Human Subjects Committee of each medical centre. Written informed consent was obtained from each patient. The demographics of the patients, including the age, sex, and ethnicity, together with the dose and duration of acid suppressive medications were recorded.

DEFINITIONS

Short segment Barrett's oesophagus (SSBO) was defined as the presence of columnar appearing mucosa in the distal oesophagus less than $3 \mathrm{~cm}$ in length with intestinal metaplasia on biopsy. Cardia intestinal metaplasia (CIM) was defined as presence of intestinal metaplasia immediately distal to the GOJ. The GOJ was defined as the pinch at the end of the tubular oesophagus coinciding with the proximal margins of the gastric folds. ${ }^{17}$
ENDOSCOPY AND BIOPSY PROTOCOL

Upper endoscopy (oesophagogastroduodenoscopy, OGD) was performed in a standard manner using either an Olympus GIF-100 or GIF-1T100 endoscope (Olympus, Lake Success, New York, USA). The appearance of the squamocolumnar junction was carefully evaluated, noted, and recorded. The presence of irregular appearing $\mathrm{Z}$ lines, tongues, or segments of columnar lining mucosa in the distal oesophagus was also recognised, noted, and biopsied.

The biopsy protocol included obtaining four quadrant biopsy samples every $2 \mathrm{~cm}$ from the circumferential appearing Barrett's epithelium in the distal oesophagus. In patients with small or irregular tongues of columnar mucosa, at least two biopsy specimens from every centimetre length in the distal oesophagus were obtained. At least four biopsy specimens were obtained from the gastric cardia. All biopsy specimens were obtained using standard biopsy forceps.

HISTOLOGICAL ANALYSIS

All biopsy specimens were fixed in formalin, embedded in paraffin wax, and stained with haematoxylin and eosin in combination with alcian blue or using periodic acid Schiff (PAS) with alcian blue at $\mathrm{pH}$ 2.5. The diagnoses of SSBO and CIM were confirmed by the presence of goblet cells (staining deep blue) in the biopsy specimens obtained from the columnar appearing distal oesophagus and the gastric cardia, respectively. Dysplasia in the biopsy specimens was recognised by the presence of cytological and architectural abnormalities and was classified as no dysplasia, indeterminate/low grade dysplasia (LGD), and high grade dysplasia (HGD) based on the criteria established for inflammatory bowel disease. ${ }^{18} \mathrm{~A}$ single pathologist at each institution reviewed all biopsy specimens with no

Table 2 Characteristics of patients with short segment Barrett's oesophagus (SSBO) and dysplasia

\begin{tabular}{|c|c|c|c|c|c|c|c|}
\hline \multirow[b]{2}{*}{ Patient no } & \multirow[b]{2}{*}{ Age/Sex } & \multirow{2}{*}{$\begin{array}{l}\text { Length of } \\
S S B O(\mathrm{~cm})\end{array}$} & \multicolumn{3}{|c|}{ Grade of dysplasia } & \multirow{2}{*}{$\begin{array}{l}\text { Total follow up } \\
\text { (months) }\end{array}$} & \multirow[b]{2}{*}{ Medical treatment } \\
\hline & & & Initial & First detection & Most recent & & \\
\hline 1 & $75 / \mathrm{M}$ & 2 & ND & HGD & Cancer & 33 & $\mathrm{H}_{2} \mathrm{RA}$ \\
\hline 2 & $54 / \mathrm{M}$ & 2 & ND & HGD & LGD & 32 & $\mathrm{H}_{2}^{2} \mathrm{RA}$ \\
\hline 3 & $69 / \mathrm{M}$ & 1 & ND & LGD & ND & 36 & Omeprazole \\
\hline 4 & $70 / \mathrm{M}$ & 2 & ND & LGD & ND & 67 & Omeprazole \\
\hline 5 & $61 / M$ & 2 & ND & LGD & ND & 36 & None \\
\hline 6 & $61 / \mathrm{M}$ & 1 & LGD & LGD & ND & 8 & $\mathrm{H}_{2} \mathrm{RA}$ \\
\hline 7 & $47 / \mathrm{M}$ & 1.5 & LGD & LGD & ND & 100 & Omeprazole \\
\hline 8 & $72 / \mathrm{M}$ & 2 & LGD & LGD & ND & 86 & Omeprazole \\
\hline 9 & $67 / M$ & 2 & LGD & LGD & ND & 41 & Omeprazole \\
\hline 10 & $56 / \mathrm{M}$ & 2 & ND & LGD & LGD & 14 & Omeprazole \\
\hline 11 & $72 / \mathrm{M}$ & 2 & LGD & LGD & ND & 54 & Omeprazole \\
\hline 12 & $44 / M$ & 1 & HGD & HGD & ND & 1 & Lansoprazole \\
\hline 13 & $70 / \mathrm{M}$ & 1 & ND & LGD & LGD & 11 & $\mathrm{H}_{2} \mathrm{RA}$ \\
\hline 14 & $53 / \mathrm{M}$ & 1 & LGD & LGD & LGD & 2 & Lansoprazole \\
\hline 15 & $50 / \mathrm{M}$ & 1 & LGD & LGD & ND & 15 & Lansoprazole \\
\hline 16 & $67 / \mathrm{M}$ & 2 & ND & LGD & ND & 18 & Lansoprazole \\
\hline 17 & $50 / \mathrm{M}$ & 1 & LGD & LGD & LGD & 3 & Lansoprazole \\
\hline 18 & $76 / \mathrm{M}$ & 1 & LGD & LGD & ND & 60 & $\mathrm{H}_{2} \mathrm{RA}$ \\
\hline 19 & $55 / \mathrm{M}$ & 1 & LGD & LGD & ND & 2 & $\mathrm{H}_{2}^{2} \mathrm{RA}$ \\
\hline 20 & $68 / \mathrm{M}$ & 2 & ND & HGD & LGD & 62 & Omeprazole \\
\hline 21 & $76 / \mathrm{M}$ & 1 & LGD & LGD & ND & 3 & $\mathrm{H}_{2} \mathrm{RA}$ \\
\hline 22 & $58 / \mathrm{M}$ & 1 & LGD & LGD & LGD & 1 & $\mathrm{H}_{2} \mathrm{RA}$ \\
\hline 23 & $68 / \mathrm{M}$ & 1 & LGD & LGD & ND & 49 & $\mathrm{H}_{2} \mathrm{RA}$ \\
\hline 24 & $49 / \mathrm{M}$ & 1 & HGD & ND & ND & 12 & $\mathrm{H}_{2} \mathrm{RA}$ \\
\hline 25 & $57 / \mathrm{M}$ & 1 & ND & LGD & ND & 8 & $\mathrm{H}_{2} \mathrm{RA}$ \\
\hline
\end{tabular}

$\mathrm{H}_{2} \mathrm{RA}, \mathrm{H}_{2}$ receptor antagonist; ND, no dysplasia; HGD, high grade dysplasia; LGD, low grade dysplasia. 


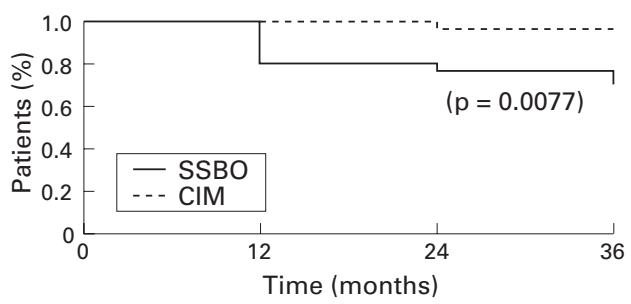

Figure 1 Dysplasia free interval. SSBO, short segment Barrett's oesophagus; CIM, cardia intestinal metaplasia.

prior knowledge of the detailed clinical history of the patients.

FOLLOW UP ENDOSCOPY

Once the diagnosis of either SSBO or CIM was established, the patients were followed prospectively and underwent upper endoscopy with biopsies every year, using the biopsy protocol described earlier. Endoscopy was performed earlier if necessary, guided by patient's symptoms or the histological presence of dysplasia. For the purpose of this study, only patients with intestinal metaplasia on two consecutive OGD examinations at least six months apart were included in the follow up analysis.

STATISTICAL ANALYSIS

Fisher's exact test and the two sample $t$ test were used to assess differences between SSBO and CIM patients for categorical and continuous variables, respectively. The log rank test was used to assess the difference in time to progression to dysplasia between SSBO and CIM patients. Three year actuarial probabilities of dysplasia development were calculated for the SSBO and CIM patients. All statistical comparisons were made at the $5 \%$ significance level.

\section{Results}

PATIENTS WITH SSBO

A total of 177 patients with SSBO was identified from both centres. The mean age of this patient group was 62 years (range 38-82). The majority $(91 \%)$ of the patients were whites (162 whites, seven African Americans, seven Hispanics, and two native Americans). The mean length of Barrett's mucosa in this group was $1.4 \mathrm{~cm}$ (range $1-2.5 \mathrm{~cm}$ ). Twenty cases of dysplasia in SSBO were detected at the initial endoscopy; including 17 patients with low
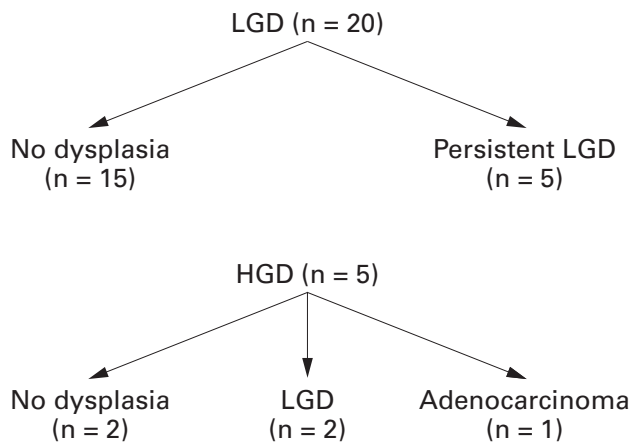

Figure 2 Outcome of dysplasia in patients with short segment Barrett's oesophagus. HGD, high grade dysplasia. LGD, low grade dysplasia grade dysplasia (LGD) and three patients with high grade dysplasia (HGD). The dysplasia prevalence in the SSBO group was $11.3 \%$ (table 1).

\section{PATIENTS WITH CIM}

Seventy six patients with CIM were identified from both centres. The mean age of this group was 67 years (range 37-81). This included $81 \%$ whites and $16 \%$ African Americans (62 whites, 12 African Americans, and two Hispanics). The CIM patients were older $(p=0.0045)$ and had a higher percentage of African Americans $(p=0.0028)$ than the SSBO patients (table 1). Only one patient was detected with dysplasia at initial endoscopy (LGD) - a prevalence of $1.3 \%$. Dysplasia prevalence was statistically higher $(p=0.0058)$ in the SSBO patients $(11.3 \%)$ than in the CIM patients $(1.3 \%)$.

FOLLOW UP OF PATIENTS WITH SSBO AND CIM A total of 78 patients with SSBO (documented intestinal metaplasia on two separate occasions) have undergone repeat endoscopy with biopsy. The mean follow up of this group was 31 months (range 8-100 months). Thirty four patients with CIM (intestinal metaplasia on two separate occasions) were followed prospectively for a mean of 24 months (range 6-80 months).

During follow up of SSBO patients, dysplasia developed in nine patients (seven LGD, two HGD). Table 2 shows the demographics and clinical features of SSBO patients with dysplasia. However, only one patient in the CIM group developed LGD. The dysplasia incidence in SSBO patients was $4.6 \%$ per year compared with $1.5 \%$ per year in CIM patients. The time to dysplasia development (fig 1) was significantly longer $(p=0.0077)$ in CIM patients compared with SSBO patients (per log rank test). Based on actuarial probabilities, only $4.2 \%$ of the CIM patients compared with $31 \%$ of the SSBO patients had developed dysplasia within three years.

Of the 29 patients with dysplasia in SSBO, 25 have been followed up. Of the five patients with SSBO and HGD (fig 2), one developed adenocarcinoma of the oesophagus over 24 months. No HGDs or cancers were detected in CIM patients during follow up.

The patient who developed dysplasia in the CIM group was a 64 year old white man who had evidence of LGD on his second endoscopy, one year after the initial diagnosis. He had evidence of Helicobacter pylori infection, did not undergo eradication therapy, and on his third endoscopy had persistent CIM but no evidence of dysplasia.

PATIENTS WITH BOTH SSBO AND CIM

From the study population, 10 patients were identified who had evidence of both lesions (SSBO and CIM). The patients in this group were all men, mean age was 63 years (range 48-73), and the mean length of Barrett's oesophagus was $1.4 \mathrm{~cm}$. Three of these 10 patients had evidence of dysplasia (two LGD, one HGD) in their oesophageal intestinal metaplasia without dysplasia in their cardia. 


\section{Discussion}

Detection of intestinal metaplasia in the distal oesophagus as well as within the gastric cardia is being reported with increasing frequency. The prevalence of SSBO has been reported to vary from $2 \%$ to $12 \%$ and that of CIM from $5 \%$ to $23 \%$ in patients undergoing routine upper endoscopy. The detection of intestinal metaplasia in the distal oesophagus (Barrett's oesophagus) potentially commits the patient to regular surveillance endoscopy with biopsy. The incidence of adenocarcinoma in patients with Barrett's oesophagus is estimated to be $30-50$ times that of the general population ${ }^{19}$ and is on the increase. However, the exact incidence of cancer in patients with SSBO is unknown, and the role of CIM as a premalignant lesion is still unclear.

This study prospectively evaluated two well defined groups of patients for the development of dysplasia and/or adenocarcinoma of the oesophagus and the gastric cardia. The prevalence and incidence of dysplasia were significantly higher in the SSBO group compared with the CIM group (11.3\% and $4.6 \%$ versus $1.3 \%$ and $1.5 \%$ respectively). Significant age, ethnic, and sex differences were also noted between the two groups. Is a comparison between these two groups valid and what are the implications of this study? SSBO and CIM patients were defined prospectively by standardised criteria at the two medical centres with a long standing interest in these entities. No consenting patients meeting the study criteria were excluded from the analysis. The demographics and dysplasia risk of patients with SSBO are different from those in CIM patients; indicating two distinct lesions with possibly separate pathophysiologies. The prevalence of dysplasia in SSBO patients in this study is comparable to that recently reported $(8 \%)$ in a group of well defined patients evaluated prospectively. ${ }^{14}$ The dysplasia risk was significantly different between SSBO and CIM patients during a relatively short follow up period but future studies with larger patient numbers and longer follow up are needed to validate these results.

Why is it important to separate SSBO from CIM and are they different entities? The distinction between intestinal metaplasia in the distal oesophagus versus the gastric cardia has not been well characterised, but recently a few studies have shed light on this issue. Recent data suggest that the aetiology of CIM is more likely to be secondary to $H$ pylori infection rather than GORD. Goldblum et al studied patients with carditis (inflammation in the gastric cardia) and CIM. ${ }^{20}$ This study found that $H$ pylori infection was closely associated with both carditis and CIM and that CIM was associated with $H$ pylori carditis as well as intestinal metaplasia in other parts of the stomach. There also seems to be a variance in the prevalence of SSBO and CIM based on different ethnic groups being evaluated. The prevalence of CIM has been shown to be equally prevalent in African Americans and whites; whereas Barrett's oesophagus is significantly more prevalent in whites. ${ }^{12}$ This difference may be secondary to a difference in pathophysiology and/or genetics.

Dias Pereira et al evaluated the prevalence of intestinal metaplasia in patients with columnar lining in the distal oesophagus and in those with a normal appearing squamocolumnar junction. ${ }^{21}$ This study included patients with SSBO and CIM. Reflux symptoms as well as erosive oesophagitis were more frequent in patients with SSBO than in CIM patients, indicating that GORD may be a possible cause of SSBO. Patients with SSBO had a higher male:female ratio (2.8:1), which is very similar to long segment or traditional Barrett's oesophagus. As opposed to SSBO, patients with CIM were predominantly women and had a lower frequency of GORD symptoms.

Recently Hackelsberger et al studied 23 patients with SSBO and 42 with CIM. ${ }^{22}$ The prevalence of CIM in the entire study group was $13.4 \%$ and was significantly associated with older age, $H$ pylori gastritis, and intestinal metaplasia elsewhere in the stomach. SSBO patients, on the other hand, were more likely to be men with evidence of GORD symptoms, erosive oesophagitis, but no association with $H$ pylori or gastric intestinal metaplasia. Thus, CIM is a relatively common finding if biopsy specimens are obtained from below a normal squamocolumnar junction and occurs mainly in older $H$ pylori infected men who also have evidence of multifocal gastric intestinal metaplasia. In a recent study, the demographics and dysplasia/cancer prevalence were studied in 45 CIM, 64 SSBO, and 40 patients with long segment Barrett's oesophagus. ${ }^{14}$ Patients with SSBO were predominantly white men with GORD symptoms. In contrast, patients with CIM were similar in sex and ethnicity to the control group and had a higher prevalence of $H$ pylori infection. Dysplasia or cancer was noted in $10 \%$ of SSBO and $6.4 \%$ of CIM patients.

There is an increasing body of evidence to suggest that intestinal metaplasia in the region of the GOJ may comprise two distinct entities: SSBO (intestinal metaplasia in the distal oesophagus); and CIM (intestinal metaplasia in the gastric cardia). The dysplasia risk, as shown by our study, is significantly different between these groups of patients. The most important finding in this study is the lower prevalence and incidence of dysplasia in CIM patients, even though they were significantly older than SSBO patients. The cancer risk associated with CIM remains unclear and although there is a potential association of CIM with cardia cancer, it seems to be very low, given that CIM is a very common finding. The concepts of SSBO and CIM are still evolving and confusion arises from the use of different terminologies, perhaps due to the difficulty in precisely defining the exact junction between the stomach and the oesophagus. This is particularly true in patients with large hiatal hernias, erosive oesophagitis, strictures, postfundoplication, or tumours at the GOJ.

The metaplasia-dysplasia-adenocarcinoma sequence is well defined for intestinal metaplasia of the distal oesophagus (Barrett's 
oesophagus), but not for intestinal metaplasia of the gastric cardia. The demographics of SSBO patients include elderly white men with associated GORD symptoms, whereas CIM may be equally prevalent in men and women, with no ethnic predilection. Patients undergoing upper endoscopy for reflux symptoms and found to have short tongues of columnar appearing mucosa should have these tongues biopsied. If these biopsies reveal intestinal metaplasia, the diagnosis of SSBO is confirmed. However, there is lack of information on the cancer risk of CIM patients and thus the taking of biopsy specimens at or below a normal appearing squamocolumnar junction is not currently recommended. Future larger prospective studies should separate SSBO from CIM in order to enhance our understanding of the pathophysiology and the malignant potential of each clinical entity. The possible role of intestinal metaplasia as a premalignant lesion of cardia cancer is not yet clear and our series indicates a very low prevalence and incidence of dysplasia within CIM. Thus, surveillance of CIM is not recommended aside from study protocols.

This work was presented as DDW Poster of Distinction at the Digestive Diseases Week, May 1999, Orlando, Florida, USA.

1 Blott WJ, Devesa SS, Kneller RW, et al. Rising incidence of adenocarcinoma of the esophagus and gastric cardia. fAMA 1991;265:1287-9.

2 Pera M, Cameron AJ, Trastek VF, et al. Increasing incidence of adenocarcinoma of the esophagus and esophagogastric junction. Gastroenterology 1993;104:510-13.

3 Blot WJ, Devesa SS, Fraumeni JF Jr. Continuing climb in rates of esophageal adenocarcinoma: An update $7 A M A$ 1993;270:1320

4 Clark G, Smyrk TC, Burdiles P, et al. Is Barrett's metaplasia the source of adenocarcinomas of the cardia? Arch Surg 1994;129:609-14. 5 Spechler SJ, Goyal RK. Barrett's esophagus. N Engl f Med 6 Sharma P, Morales TG, Sampliner RE. Short segment Barnition and of endoscopic criteria. Am $\mathcal{F}$ Gastroenterol 1998; 93:1033-6.

7 Weston AP, Krmpotich P, Makdisi WF, et al. Short segment Barrett's esophagus: clinical and histological features, associated endoscopic findings, and association with gastric intestinal metaplasia. Am $\mathcal{F}$ Gastroenterol 1996;91:981-6.

8 Johnston MH, Hammond AS, Laskin W, et al. The prevalence and clinical characteristics of short segments of specialized intestinal metaplasia in the distal esophagus on routine endoscopy. Am f Gastroenterol 1996;91:1507-11.

9 Spechler SJ, Zeroogian JM, Antonioli DA, et al. Prevalence of metaplasia at the gastro-oesophageal junction. Lancet 1994;344:1533-6.

10 Weston AP, Krmpotich PT, Cherian R, et al. Prospective long-term endoscopic and histological follow-up of short segment Barrett's esophagus: comparison with traditional long segment Barrett's esophagus. Am f Gastroenterol 1997; 92:407-13.

11 Morales TG, Sampliner RE, Bhattacharyya A. Intestinal metaplasia of the gastric cardia. Am $\mathcal{F}$ Gastroenterol 1997;92:414-18.

12 Chalasani N, Wo JM, Hunter JG, et al. Significance of intestinal metaplasia in different areas of esophagus including esophagogastric junction. Dig Dis Sci 1997;42:603-7.

13 Sharma P, Morales TG, Bhattacharyya A, et al. Dysplasia in short-segment Barrett's esophagus: A prospective 3-year follow-up. Am f Gastroenterol 1997;92:2012-16.

14 Hirota WK, Loughney TM, Lazas DJ, et al. Specialized intestinal metaplasia, dysplasia and cancer of the esophagus and esophagogastric junction: prevalence and clinical data. Gastroenterology 1999;116:277-85.

15 Hamilton SR, Smith R, Cameron JL. Prevalence and characteristics of Barrett esophagus in patients with adenocarcinoma of the esophagus or esophagogastric junction. Hum Pathol 1988;19:942-8.

16 Schnell TG, Sontag SJ, Chejfec G. Adenocarcinomas arising in tongues or short segments of Barrett's esophagus. arising in tongues or short segm

17 McClave SA, Boyce HW, Gottfried MR. Early diagnosis of columnar lined esophagus: a new endoscopic criterion. columnar lined esophagus: a new

18 Riddell RH, Goldman H, Ranshoff DF, et al. Dysplasia in inflammatory bowel disease: standardized classification with provisional clinical implication. Hum Pathol 1983;14: 931-68.

19 Reid BJ. Barrett's esophagus and esophageal adenocarcinoma. Gastroenterol Clin North Am 1991;20:817-34.

20 Goldblum JR, Vicari JJ, Falk GW, et al. Inflammation and intestinal metaplasia of the gastric cardia: the role of gastroesophageal reflux and $\mathrm{H}$. pylori infection. Gastroenterology 1998;114:633-9.

21 Dias Pereira A, Suspiro A, Chaves P, et al. Short segments of Barrett's epithelium and intestinal metaplasia in normal appearing oesophagogastric junctions; the same or two different entities? Gut 1998;42:659-62.

22 Hackelsberger A, Gunther T, Schultze V, et al. Intestinal metaplasia at the gastro-oesophageal junction: Helicobacter pylori gastritis or gastro-oesophageal reflux disease? bacter pylori gastritis
Gut 1998;43:17-21. 